\title{
Fistule colovésicale et diverticulose sigmoïdienne colique
}

\section{Colovesical fistula and diverticulitis of the sigmoid colon}

\author{
J. Gouverneur $\cdot$ A. Pierron · V. Ruche \\ Reçu le 25 janvier 2018; accepté le 30 mars 2018 \\ (C) SFMU et Lavoisier SAS 2018
}

Une patiente âgée de 87 ans consulte au service d'accueil des urgences pour prise en charge de troubles fonctionnels urinaires évoluant depuis une dizaine de jours avec majoration de la symptomatologie clinique (pollakiurie, dysurie, brûlures mictionnelles) depuis ces trois derniers jours. Elle a consulté auprès de son médecin traitant dès les premiers symptômes, et un traitement par fluoroquinolone a été prescrit devant le diagnostic d'infection urinaire basse. Depuis trois jours, les signes fonctionnels urinaires ont récidivé, et les urines sont beaucoup plus foncées que par rapport à d'habitude. La patiente a apporté un échantillon de ces urines : urines de couleur marron avec dépôts dans le flacon très évocateurs de fécalurie (Fig. 1A). Les antécédents médicaux sont une hypertension artérielle et une dyslipidémie ; sur le plan chirurgical : appendicectomie et hystérectomie. La patiente est en excellent état général. Les constantes vitales sont les suivantes : tension artérielle à $123 / 76 \mathrm{mmHg}$, température à $36,3{ }^{\circ} \mathrm{C}$, pouls à $82 / \mathrm{min}$, $\mathrm{SpO}_{2}$ à $98 \%$ en air ambiant. L'examen clinique est sans particularité ; l'abdomen est parfaitement souple et indolore en particulier en fosse iliaque gauche. La biologie sanguine montre : une hyperleucocytose à $12100 / \mathrm{mm}^{3}$ dont $8216 \%$ de polynucléaires neutrophiles, une CRP à $65 \mathrm{mg} / 1$ et une fonction rénale normale. La tomodensitométrie (TDM) abdominopelvienne avec injection de produit de contraste met en évidence une collection hydroaérique périsigmoïdienne se fistulisant au dôme vésical avec une importante infiltration périlésionnelle et des bulles d'air au sein de la vessie (Fig. 1B). Cette image est compatible avec une collection abcédée fistulisée dans la vessie sur sigmoïdite diverticulaire. La patiente bénéficie alors d'une antibiothérapie par voie intraveineuse ainsi que d'une sigmoïdectomie avec anastomose colorectale et suture vési-

\footnotetext{
J. Gouverneur · A. Pierron · V. Ruche $(\square)$

Service des urgences,

centre hospitalier Geneviève-de-Gaulle-Anthonioz

rue du Dr-Schweitzer, F-52100 Saint-Dizier, France

e-mail : valerie.ruche@ch-santdizier.fr
}

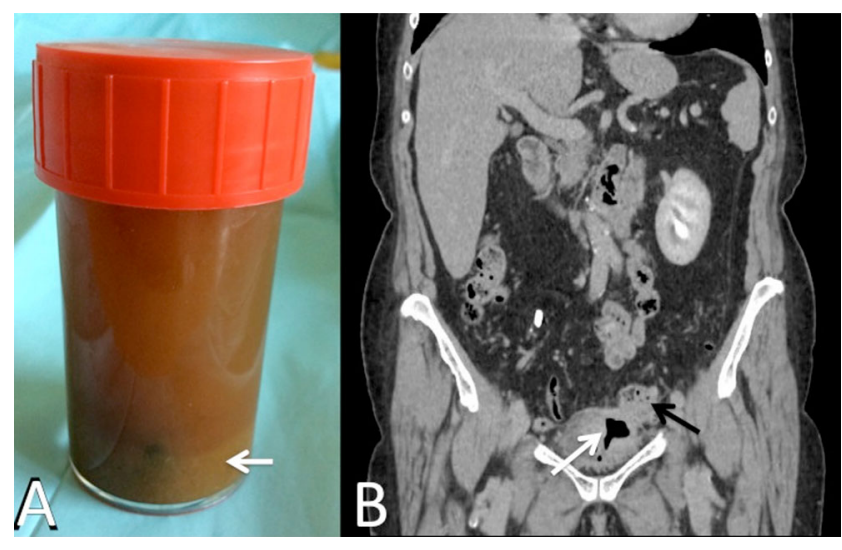

Fig. 1 Fistule colovésicale et diverticulose sigmoïdienne colique. A. Fécalurie avec sédiments de selles (flèche blanche). B. TDM abdominale injectée, coupe frontale : bulles d'air dans la vessie (flèche blanche), collection hydro-aérique se fistulisant au dôme vésical (flèche noire)

cale. Les suites opératoires sont simples, et la patiente est sortante dix jours plus tard.

La fistule colovésicale est un événement rare de la maladie diverticulaire colique. Cette dernière est la cause la plus fréquente de fistules entérovésicales devant les cancers et les colites inflammatoires ou radiques. Les signes urinaires (infections urinaires récidivantes, pollakiurie, pneumaturie) sont souvent au-devant du tableau clinique de la fistule entérovésicale. La fécalurie, plus rare, est pathognomonique d'une fistule colovésicale [1]. La TDM abdominopelvienne avec injection de produit de contraste constitue l'examen diagnostique de référence, permettant de mettre en évidence une bulle d'air intravésicale (signe le plus constant, mais non contributif en cas de sondage urinaire récent), des diverticules sigmoïdiens (retrouvés dans $90 \%$ des cas) ; la communication est rarement mise en évidence. Le traitement est chirurgical. De nombreuses équipes préconisent la chirurgie en deux temps (selon les constatations peropératoires) : intervention de Hartmann avec colostomie de décharge 
temporaire (fermeture de l'iléostomie quelques semaines plus tard) [2] ; au niveau du versant vésical, suture vésicale, mais une cystectomie partielle peut s'avérer nécessaire. Toute découverte de fécalurie doit faire évoquer le diagnostic de fistule entérovésicale et nécessite des explorations complémentaires afin de préciser l'origine de cette fistule.

\section{Références}

1. Long JA, Descotes JL, Rambeaud JJ (2009) Fistules vésicales. EMC (Elsevier Masson SAS, Paris) Urologie 18-215-A-10

2. Smeenk RM, Plaisier PW, van der Hoeven JAB, et al (2012) Outcome of surgery for colovesical and colovaginal fistulas of diverticular origin in 40 patients. J Gastrointest Surg 16:1559-65 